Article

\title{
Development and Characterization of a Large Mutant Population of a Rice Variety Katy for Functional Genomics Studies and Breeding
}

\author{
Yulin Jia ${ }^{1, *}$, Zhonghua Wang ${ }^{1,2}$, Melissa H. Jia ${ }^{1}$, J. Neil Rutger ${ }^{\dagger}$, \\ Karen A. K. Moldenhauer ${ }^{3}$ \\ 1 Dale Bumpers National Rice Research Center, United States Department of \\ Agriculture Agricultural Research Service (USDA ARS), Stuttgart, \\ AR 72160, USA \\ 2 Institute of Biotechnology, Zhejiang Wanli University, Ningbo, \\ Zhejiang 315100, China \\ 3 Rice Research and Extension Center, University of Arkansas, Stuttgart, \\ AR 72160, USA \\ $\dagger$ USDA ARS retired \\ * Correspondence: Yulin Jia, Email: Yulin.jia@ars.usda.gov; \\ Tel.: +1-(870)-672-6107.
}

\begin{abstract}
The availability of a large mutant population is an important genetic resource for functional genomic studies and breeding. Katy, a publicly available long grain tropical japonica rice variety with an excellent package of disease resistance and suitable agronomic traits, was used to develop a large mutant population. A combination of ethyl methane sulfonate (EMS), fast neutron (FN), and gamma irradiation $\left({ }^{60} \mathrm{Co}\right)$ treatments were applied at varying dosages to develop the population. Approximately 7500 rice seeds were each treated with EMS $(0.4 \%, 0.8 \%$, and $1.2 \%$ ) and ${ }^{60} \mathrm{Co}$ (200 Gy), and approximately 10,000 rice seeds were exposed to $7.7 \mathrm{~Gy}, 26.3 \mathrm{~Gy}$, and $49.4 \mathrm{~Gy}$ dosages of FN. Mutation effects were initially evaluated at the $\mathrm{M}_{2}$ generation by assessing chlorophyll biosynthesis deficiencies. The mutation effects of each line were detected

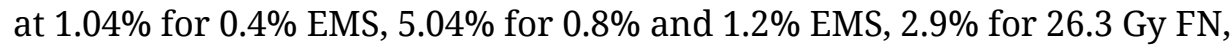
$3.2 \%$ for $49.4 \mathrm{~Gy} \mathrm{FN}$, and $5.04 \%$ for ${ }^{60} \mathrm{Co} 200 \mathrm{~Gy}$ for the $\mathrm{M}_{2}$ population. After seeds were advanced to $\mathrm{M}_{4}$ using a single seed breeding strategy, the genotype identity of 189 randomly selected lines was verified with nine simple sequence repeat markers and 96 randomly selected lines were evaluated for mutant morphological phenotypes. The analysis uncovered slightly higher frequencies of morphological mutants at $\mathrm{M}_{4}$ than was observed in the $\mathrm{M}_{2}$ generation. These findings suggest that the Katy putative mutant population consisting of 23,558 individuals is a potential asset for rice functional genomics studies and breeding.
\end{abstract}

\section{G Open Access}

Received: 26 June 2019

Accepted: 15 August 2019

Published: 19 August 2019

Copyright $\odot 2019$ by the author(s). Licensee Hapres, London, United Kingdom. This is an open access article distributed under the terms and conditions of Creative Commons Attribution 4.0 International License.

KEYWORDS: breeding; functional genomics; Katy rice; mutagen; morphological mutants; Oryza sativa 


\section{ABBREVIATIONS}

EMS, ethyl methane sulfonate; FN, fast neutron; ${ }^{60} \mathrm{Co}$, Cobalt 60; GSOR, genetic stocks Oryza; SSR, simple sequence repeat

\section{INTRODUCTION}

Rice (Oryza sativa) is one of the most important food crops that provide essential calories to more than 3.5 billion people. Continued improvement of yield, quality, and stress tolerance is extremely important for food security and world peace. The genome of rice is the smallest among the major monocot crops and has been fully sequenced and assembled. The rice genome is $370 \mathrm{Mb}$ in size and encodes over 50,000 protein coding genes [1]. Easy methods of transformation and abundant transcription profiles have made rice an excellent model crop for functional genomics [2]. Thus far, biological functions of about 2000 rice genes from genes for high-yield to disease resistance [1-3] have been determined, most of which with a map-based cloning strategy [3]. While map-based cloning has been proven to be one of the most successful methods of studying gene function, it requires contrasting the phenotype and genotype of both parents which are often not available, particularly for genes contributing minor phenotypic effect and genes involved in multiple biological functions via epigenetic mechanisms [4-6].

One of the major hurdles in studying the biological functions of rice genes is the availability of genetic variation among existing rice germplasms. Natural variation of rice has been extremely limited and unbalanced due to domestication and extensive high-yielding rice breeding and selections [7]. Mutagenesis is the process of inducing artificial genetic variation by chemical and physical mutagens to link altered sequence information to corresponding phenotypic traits. The chemical mutagen ethyl methane sulfonate (EMS) and irradiation by fast neutron (FN) and gamma irritation $\left({ }^{60} \mathrm{Co}\right)$ have been widely used to induce many phenotypic variations in rice [8-12]. Deletions and point mutations induced by various mutagens increase the probability of discovering desirable traits in the genome that were suppressed during selective breeding due to linkage drag [13]. Next-generation sequencing technology, including Targeting Induced Local Lesions IN Genomes (TILLING)[14] and Deletagene [12], for each type of mutagen enables the manageable discovery and identification of candidate genes.

The most prevalently used chemical mutagen for rice is EMS due to its potency and the simple method of application [9]. Studies indicate that increased concentration of EMS eventually leads to a decrease in germination, root length, and seedling height in the MR219 variety beginning at $0.25 \%$ and $0.50 \%$ [9]. Therefore, higher dosages will likely increase plant mortality rates within the mutagenized population. ${ }^{60} \mathrm{Co}$ is also a commonly used mutagen and was effective in producing small deletions from 1 and several $10 \mathrm{bp}$ or around $130 \mathrm{kbp}$ in rice [11]. ${ }^{60} \mathrm{Co}$ 
tends to frequently induce small deletions along with a few large deletions and point mutations, and is lethal for biological tissue at high dosages. The use of FN mutagenesis is a relatively new method in forward genetics that employs neutron bombardment [12]. The sequencing gene knockout technique Deletagene has also been developed in rice to detect small mutations as well as deletions of up to three homologous genes [12]. This method increases the probability of discovering mutations in comparison to some other techniques. It is predicted that FN mutagenesis will generate the most morphological mutants even at low dosages because it is a highly penetrative form of radiation and will cause less lethal deletions.

Morphological mutations and dwarfism are easily identifiable visible phenotypes within mutagenized rice populations. Morphological mutants are a good indicator of any other genetic changes in a mutant population consisting of multiple dosages, different mutagens, and easily identifiable phenotypes [10]. Katy rice is a publicly available high-yielding long grain tropical japonica variety that is well adapted to the southern USA and contains key resistance genes (Pi-ta, Pi-ta2, Ptr, and Pi-ks) to rice blast disease caused by the fungus Magnaporthe oryzae and moderate sheath blight resistance genes caused by the fungus Rhizoctonia solani [15-18]. Katy rice is, therefore, a key target cultivar for modern rice breeding programs. Consequentially, a dozen rice cultivars and several mapping populations were released with Katy as a parent [17].

The objectives of this study were to develop mutagenized Katy rice populations comparing the effectiveness of FN, EMS, and ${ }^{60} \mathrm{Co}$ mutagenesis at the $\mathrm{M}_{2}$ and $\mathrm{M}_{4}$ generations. The genetic background of the population was verified with a set of diagnostic single sequence repeat DNA (SSR) markers. The mutation effect was estimated using visible traits, chlorophyll deficiency, dwarfism, and curly leaf phenotypes using healthy mutagen treated rice as an internal control.

\section{MATERIALS AND METHODS}

\section{Plant Materials}

Katy (Reg no78, PI 527707), developed by University of Arkansas Rice Research and Extension Center (UA RREC), Stuttgart, Arkansas, USA, is a publicly available rice blast resistant, mid-season, acceptable yield long grain cultivar. Approximately $1 \mathrm{~kg}$ of rice was generously provided by Karen Moldenhauer, UA RREC [15].

\section{Mutagen Treatment}

The first subset of 15,192 putative mutants (GSOR 600000-615192) were treated with 7.7 Gy, 26.3 Gy, and 49.4 Gy fast neutron (FN) at Oak Ridge National Laboratory, Oak Ridge, Tennessee, USA by Roger Martin. The second subset of 4879 putative mutants (GSOR 616001-620880) were treated with $0.4 \%, 0.8 \%$, and $1.2 \%$ ethyl methane sulfonate (EMS). For each treatment, $150 \mathrm{~g}$ of Katy rice was used. EMS treatment was as described in 
$\mathrm{Hu}$ and Rutger [19] and performed at USDA ARS Dale Bumpers National Rice Research Center in 2001. Specifically, approximately 7500 seeds of Katy were treated with $0.8 \%(\mathrm{v} / \mathrm{v})$ EMS in 2001. The $\mathrm{M}_{1}$ generation was planted at Stuttgart, Arkansas, USA in the spring of 2001, and 1434 panicles were randomly harvested that fall. Five to seven seeds from each $\mathrm{M}_{1}$ panicle were germinated in a $1.5-\mathrm{mL}$ microcentrifuge tube in a $30^{\circ} \mathrm{C}$ dark incubator for 3 days. Germinated seeds were planted in a small pot in the greenhouse and transferred for subsequent seed amplification under field conditions. The third subset of 2769 putative mutants (GSOR 620900623669) were treated with ${ }^{60} \mathrm{Co}$ at the rate of $200 \mathrm{~Gy}$ at Florida Accelerator Services and Technology, 1911 SW 34th street, Gainesville, Florida, USA by Mr. Burrell in 2002. A total of 2769 panicles were harvested from $2769 \mathrm{M}_{1}$ plants and were subsequently amplified to $\mathrm{M}_{4}$ by a single seed breeding method in Stuttgart, Arkansas, USA from 2004 to 2007 under greenhouse and field conditions.

\section{Growth and Evaluation of the Mutation Rate Using Morphological Mutations}

A total of 33,653 randomly selected $\mathrm{M}_{2}$ seeds derived from $3300 \mathrm{M}_{1}$ plants from different mutagens and dosages were evaluated under greenhouse conditions in 2002. Seeds were sown in trays containing 96 well inserts. Two to three seedlings per well were grown with $30-40 \mathrm{~g}$ of coarse electrically sterilized silt loam soil from Arkansas facilitating root growth. The plants were scored at the 3 to 4 leaf stages. Healthy mutagen treated Katy rice with normal morphological traits and untreated Katy rice were used as the control. Pots that contained morphological mutations in all seeds within a rice line were scored according to their distinctive phenotype. The seeds were carried in a cart and placed adjacent to one another inside a greenhouse at around $30{ }^{\circ} \mathrm{C}$ : the temperature most suitable for rice germination and growth. The plants were sub-irrigated with around $2 \mathrm{~L}$ per plot and $30 \mathrm{~g}$ of fertilizer (Osmocote) were placed inside each plot. The seeds were watered with the same amount every 2 or 3 days depending on the amount of water left inside the plot.

The first subset of 15,192 putative mutants were treated by FN with 7.7 Gy, 26.3 Gy, and 49.4 Gy. A total of 15,192 $\mathrm{M}_{1}$ panicles were harvested from $15,192 \mathrm{M}_{1}$ and were subsequently amplified to $\mathrm{M}_{4}$ by single seed breeding method in Stuttgart, AR, from 2003 to 2006. An evaluation of 190 randomly selected plants from the entire population in 2006 verified that they were derived from Katy based on 9 diagnostics simple sequence repeat markers (Supplementary Table S1) (GSOR 600001-615192). The second subset of mutants were treated with $0.4 \%, 0.8 \%$, and $1.2 \%$ EMS. A total of 4880 panicles were harvested from $4896 \mathrm{M}_{1}$ and were subsequently amplified to $\mathrm{M}_{4}$ by single seed breeding method in Stuttgart, AR from 2004 to 2007 (GSOR 616001-620880). The third subset of mutants was treated with gamma irradiation at the rate of $200 \mathrm{~Gy}$. A total of 2769 panicles were harvested from $2769 \mathrm{M}_{1}$ and were subsequently amplified to $\mathrm{M}_{4}$ by single 
seed breeding method in Stuttgart, AR from 2004 to 2007 (GSOR 620900623669). Mutation effects of $\mathrm{M}_{4}$ seedlings were evaluated as described below from 2017 to 2018 (Table 1).

Table 1. A large mutant population of Katy rice treated with different mutagens and dosages.

\begin{tabular}{lllll}
\hline Mutagen & GSOR & Dosage & $\mathbf{M}_{\mathbf{4}}$ lines & Total lines \\
\hline Fast neutron & $600000-607000$ & $7.7 \mathrm{~Gy}$ & 7000 & \\
Fast neutron & $607001-614849$ & $26.3 \mathrm{~Gy}$ & 7849 & \\
Fast neutron & $614850-615192$ & $49.4 \mathrm{~Gy}$ & 343 & 15,192 \\
\hline EMS & $616001-620880$ & $0.4 \%(\mathrm{v} / \mathrm{v})$ & 1538 & \\
EMS & & $0.8 \%(\mathrm{v} / \mathrm{v})$ & 1434 & 4896 \\
EMS & & $1.2 \%(\mathrm{v} / \mathrm{v})$ & 1908 & 2769 \\
\hline${ }^{60}$ Co & $620900-623669$ & $200 \mathrm{~Gy}$ & 2769 & 23,558 \\
Sum & & & \\
\hline
\end{tabular}

To calculate the mutation effect of $\mathrm{M}_{4}$ seedlings for chlorophyll biosynthesis deficiencies, eight seeds for each of the $96 \mathrm{M}_{4}$ lines treated with either 0.4\% EMS, 1.2\% EMS, 7.7 Gy FN, 49.4 Gy FN, or 200 Gy ${ }^{60}$ Co were requested from USDA ARS (GSOR; www.ars.usda.gov/GSOR). Three independent trials were performed from September 2017 to April 2018 under greenhouse conditions. Trial 1: each $\mathrm{M}_{4}$ Katy rice progeny line was labelled according to the type of mutagen and GSOR number resulting in a total of 96 rice lines per type and concentration of mutagen. Four seeds of each GSOR line were placed into the individual pots and covered lightly with fine silt loam soil. Trial 2: new silt loam soil was ordered and placed into 15 plots in the same manner with 32 larger pots that would allow root growth for a longer duration. The project was repeated with the original seeds in nine plots to observe whether the same morphological mutant can be identified again for each line despite health defects. Seeds from the 1.2\% EMS lines (had one plot with 33 pots) and the 49.4 Gy FN lines were placed in three plots for each trial. Plastic labels were placed inside each pot to identify each line. Various $\mathrm{pH}$ tests were conducted using $\mathrm{pH}$ strips with a range of 0-14.0, 6.0-7.7, and 4.5-10 pH. Fertilizer and iron chelate, Sequestrene $330 \mathrm{Fe}$ (BASF, Lakeland, FL, USA), were added to correct soil conditions, prevent iron deficiencies, and reduce abnormality of rice seedlings. Mutants were scored using the same time-period as the first trial. Trial 3: the final set of higher concentrations was repeated using the same soil and the same procedure.

\section{Genotyping Analysis Using Diagnostic Simple Sequence Repeat (SSR) Markers}

\section{Seedling growth and DNA extraction}

Rice seedlings ( 5 per lines) were grown to the 3 to 4 leaf stages under greenhouse conditions for DNA extraction. DNA was extracted utilizing a 
rapid DNA extraction described by Xin et al. [20] except $70 \mu \mathrm{L}$ of buffer A and buffer B was used and the DNA was diluted 1:4 in TE.

\section{Marker selection and analysis}

Nine high polymorphism information content value simple sequence repeat (SSRs) markers were selected from Garris et al. [21] and Agrama et $a l$. [22] to genotype 189 Katy mutant lines with Katy rice as the wild type control (Supplementary Table S1). PCRs were performed in $25 \mu \mathrm{L}$ reaction volumes containing $10 \mathrm{mM}$ Tris- $\mathrm{HCl} \mathrm{pH} \mathrm{8.3,50} \mathrm{mM} \mathrm{KCl,} 2.5 \mathrm{mM} \mathrm{MgCl}_{2}$, $300 \mathrm{nM}$ each primer, 1 unit of Taq DNA polymerase (Promega, Madison, WI, USA) and $4 \mu \mathrm{L}$ template DNA. For each SSR marker, the forward primer was labeled with fluorescent dyes, 6FAM, NED, and Hex, from Applied Biosystems (Foster City, CA, USA) or Integrated DNA Technologies (Coralville, IA, USA). Reverse primers were unlabeled. PCR amplifications were performed using MJ Research Tetrad thermocyclers (Waltham, MA, USA) under the following conditions:(1) initial denaturation at $94{ }^{\circ} \mathrm{C}$ for $5 \mathrm{~min}$; (2) 35 cycles of $94^{\circ} \mathrm{C}$ for $30 \mathrm{~s}, 55-67^{\circ} \mathrm{C}$ for $30 \mathrm{~s}$, and $72{ }^{\circ} \mathrm{C}$ for $1 \mathrm{~min}$; (3) $5 \mathrm{~min}$ final extension at $72^{\circ} \mathrm{C}$. Three PCR products were combined based on the color and size of amplified PCR products along with $9 \mu \mathrm{L}$ of Rox labelled size standard (Applied Biosystems, Foster City, CA, USA). The PCR products were separated on an ABI 3730 DNA Analyzer according to the manufacturer's protocol (Applied Biosystems, Foster City, CA, USA). SSR fragment size was estimated using Gene Mapper ${ }^{\circledR}$ software version 3.7 (Life Technologies Corp., Carlsbad, CA, USA).

\section{RESULTS}

\section{Development of a Large Saturated Katy Mutant Population}

A total of 15,192 $\mathrm{M}_{1}$ panicles were harvested from a FN treated $\mathrm{M}_{1}$ population and were subsequently amplified to the $\mathrm{M}_{4}$ generation by a single seed breeding method in Stuttgart, AR, from 2003 to 2006 (Figure 1).

An evaluation of 190 randomly selected plants from the entire population in 2007 verified that a vast majority of putative Katy rice lines was derived from Katy based on nine diagnostic SSRs (Supplementary Table S1).

A total of 4879 panicles were harvested from 4896 EMS treated $\mathrm{M}_{1}$ population and were subsequently amplified to $\mathrm{M}_{4}$ by a single seed breeding method in Stuttgart, AR from 2004 to 2007. A total of 2769 panicles were harvested from $2769{ }^{60} \mathrm{Co} \mathrm{M}_{1}$ and were subsequently amplified to the $\mathrm{M}_{4}$ population by a single seed breeding method in Stuttgart, AR from 2004 to 2007 under greenhouse and field conditions. Together, these materials represent a large saturated Katy mutant population consisting of $23,558 \mathrm{M}_{4}$ lines (Table 1). 


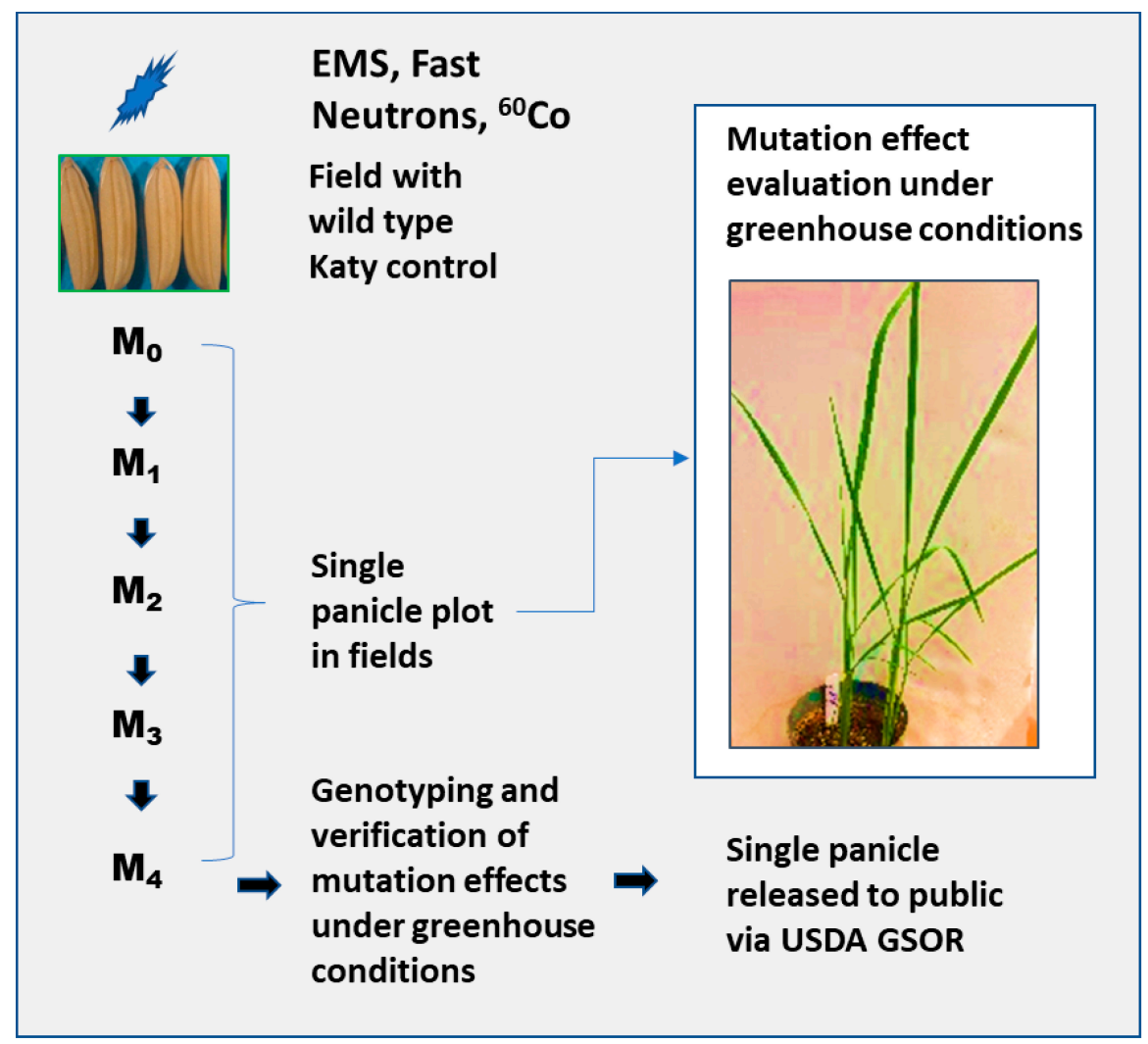

Figure 1. Schematic presentation of procedure of Katy mutant population development and characterization. Katy seeds were treated with different mutagens.

\section{Evaluation of Mutation Rate Using Deficiency in Chlorophyll Biosynthesis}

To evaluate the mutation rate of randomly selected $\mathrm{M}_{2} / \mathrm{M}_{1}$, seedlings were evaluated under greenhouse conditions from 2002 to 2003 (Table 2). No emergence differences were noted for all observed mutants in $\mathrm{M}_{2}$.

Table 2. Estimation of the mutation rate using deficiency in chlorophyll biosynthesis in $\mathrm{M}_{2}$.

\begin{tabular}{llll}
\hline Mutagen & Dosage ${ }^{1}$ & $\mathbf{M}_{\mathbf{2}} / \mathbf{M}_{\mathbf{1}}$ seedling $^{2}$ & Defect rate (\%) $^{\mathbf{3}}$ \\
\hline EMS & $0.4 \%$ & $472 / 50$ & 1.06 \\
EMS & $0.8 \%$ & $398 / 40$ & 5.04 \\
EMS & $1.2 \%$ & $436 / 40$ & 5.04 \\
Fast neutrons & $26.3 \mathrm{~Gy}$ & $1296 / 130$ & 2.90 \\
Fast neutrons & $49.4 \mathrm{~Gy}$ & $343 / 40$ & 3.20 \\
${ }^{60}$ Co & $200 \mathrm{~Gy}$ & $30,090 / 5000$ & 5.04 \\
Total & & $33,653 / 3300$ & \\
\hline
\end{tabular}

$1 \%$ refers the percentage of active gradient of EMS (v/v); Gy stands for gray (1 joule $\mathrm{kg}^{-1}$ of target specimen);

${ }^{2}$ Number of $\mathrm{M}_{2}$ seedlings and number of $\mathrm{M}_{1}$ counted; ${ }^{3}$ Number of yellow and albino seedlings over the total $\mathrm{M}_{2}$ seedlings counted.

All mutagens had some effect in causing visible chlorophyll biosynthesis deficiencies. Among them, EMS $1.2 \%$ and ${ }^{60} \mathrm{Co}$ produced the highest mutation frequencies. After the seeds were advanced from the $\mathrm{M}_{2}$ 
to the $\mathrm{M}_{4}$ generation the mutation effects of most of mutagens and dosages at the $\mathrm{M}_{4}$ were examined again (Figure 1). Initially, only a few yellow plants were identified. The experiment was then repeated at different time intervals and different chlorophyll biosynthesis mutations were identified that were expressed as yellow and albinism (Figure 2).

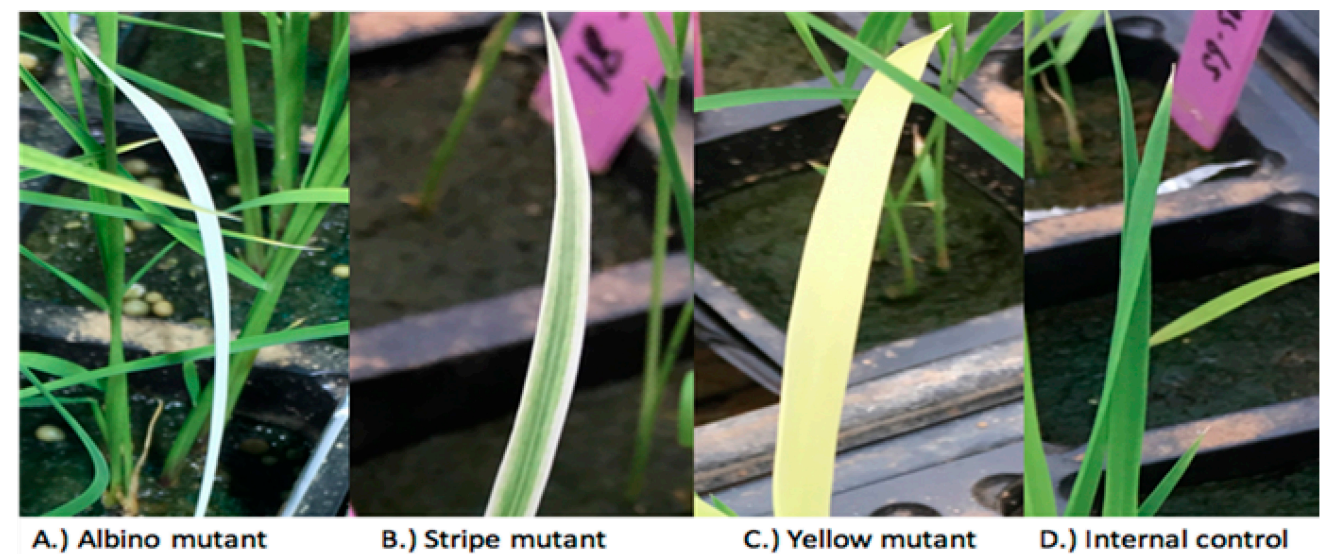

Figure 2. Examples of chlorophyll biosynthesis morphological mutant phenotypes that were observed in the mutagen-exposed populations. Plants were scored biweekly after the two leaf stage for approximately 8 weeks. If all plants per GSOR line have the same phenotype then this GSOR is called a viable homozygous mutant (Germinal mutants were shown).

No emergence differences were noted for all observed mutants in $\mathrm{M}_{4}$.

In the second trial and with the higher concentration FN and EMS samples, all rice seedlings were noticeably healthier using newly ordered silt loam soil. There were eight different putative mutants in the $0.4 \% \mathrm{EMS}$, nine in the $7.7 \mathrm{~Gy} \mathrm{FN}$, and six in the $200 \mathrm{~Gy}{ }^{60} \mathrm{Co}$ population. There were 12 different mutants in 1.2\% EMS and 11 in FN 49.4 Gy. The total number of independent mutant lines divided by the total number of rice lines grown provided the estimated mutation frequency (Table 3).

Table 3. Verification of mutation effect using deficiency in chlorphyll biosynthesis in $\mathrm{M}_{4}$.

\begin{tabular}{lllll}
\hline Mutagen & Dosage $^{\mathbf{1}}$ & $\mathbf{M}_{\mathbf{4}}$ seedlings $^{{ }^{2}}$ & $\begin{array}{l}\text { No. of } \\
\text { morphological } \\
\text { mutants }^{3}\end{array}$ & Defect rate (\%) $^{\mathbf{4}^{*}}$ \\
\hline EMS & $0.4 \%$ & 96 & 8 & 8.34 \\
EMS & $1.2 \%$ & 96 & 12 & 12.5 \\
Fast neutrons & $7.7 \mathrm{~Gy}$ & 96 & 9 & 9.4 \\
Fast neutrons & $49.4 \mathrm{~Gy}$ & 96 & 11 & 11.5 \\
${ }^{60}$ Co & $200 \mathrm{~Gy}$ & 96 & 6 & 6.3 \\
\hline
\end{tabular}

$1 \%$ refers the percentage of active gradient of EMS (v/v); Gy stands for gray (1 joule $\mathrm{kg}^{-1}$ of target specimen); ${ }^{2}$ Total number of lines (2 to 4 seedlings/line were observed in different seasons from September 2017 to April 2018); ${ }^{3}$ Number of yellow, abilno, stripe albino and dwarf seedlings observed for each mutagen/dosage; ${ }^{4}$ Number of yellow and albino seedlings over the total $\mathrm{M}_{4}$ seedlings/line counted. 
Interestingly, FN 89 GSOR line 616089 was yellow in the first trial but was albino in the second trial (data not shown). The percentages of morphological mutants versus healthy mutagen-treated rice ranged from $6.3 \%$ for the $200 \mathrm{~Gy}{ }^{60} \mathrm{Co}$ treated population to $12.5 \%$ for the $1.2 \%$ EMS treated population (Table 3).

We found that higher dosages of mutagen exposure generated more viable morphological mutants than the lower concentrations as expected. Collectively, EMS produced the most morphological mutants. In both trials, most morphological mutants were chlorophyll biosynthesis mutants likely because the rice genome contains a substantial number of genes associated with chlorophyll biosynthesis. This further validates the method used to score mutation frequencies.

\section{DISCUSSION}

It is known that a plant gene involved in disease resistance may also be involved in morphology or yield-related traits [23]. Similarly, many genes may be redundantly involved in the expression of one single trait as a failsafe [3]. The deletion of genes to characterize disease resistance genes has a degree of unreliability because the presence of a redundant gene may maintain the cultivars resistance [3,23]. Therefore, in genomic science it is important to identify all genes within a genome and elucidate their roles in the organism. Likewise, there are many redundant morphological genes, but the number of genes linked to the phenotype exceeds the number of resistance genes.

It is advisable to use multiple mutagens since each mutagen generates different types of mutations. EMS is simple and potent [9], ${ }^{60} \mathrm{Co}$ was effective for rice [11], FN generated more types of mutations [14]. In the present study, we created and released a large putative mutant population using the tropical japonica rice variety Katy for disease resistance, adapted yield, and acceptable quality. Using this putative mutant population, we identified a lesion mimic mutant created by EMS [24] and blast susceptible mutant M2354 created by fast neutrons [18]. M2354 helped clone an atypical helper Ptr for classical plant disease resistance gene. The Ptr gene independently confers broad spectrum blast resistance demonstrating a sophisticated plant immune response [3]. The extent of mutation in rice genome was examined with morphological mutants using $\mathrm{M}_{2} / \mathrm{M}_{1}$ seedlings and largely verified using randomly selected $\mathrm{M}_{4}$ lines, and the population was released to the public through USDA ARS GSOR (www.ars.usda.gov/GSOR). Together, we suggest that this large putative mutant population will be a valuable resource for rice functional genomics studies and breeding.

Greenhouse conditions at USDA Dale Bumpers National Rice Research Center are not optimal for the production of healthy rice in winter. However, the greenhouse is useful for the evaluation of the mutational effect of rice seedlings because the seedlings are placed under highly controlled conditions. The addition of equal amounts of fertilizer, the iron 
additive, and water contributed to the additional control of environmental parameters. Under greenhouse conditions, the visible morphological mutation rate ranged from $1.06 \%$ to $5.04 \%$ in the $\mathrm{M}_{2}$ generation and $6.3 \%$ to $12.5 \%$ in the $\mathrm{M}_{4}$ populations of Katy rice treated with EMS, FN, and ${ }^{60} \mathrm{Co}$, suggesting that the mutation effects are influenced by the type of mutagen and the dosage of exposure as expected (Tables 2 and 3). It is important to note that FN mutation effects were lower than those of EMS (5\%) and ${ }^{60} \mathrm{Co}$ (5\%) when $\mathrm{M}_{2}$ seedlings were evaluated (Table 2). In contrast, the chlorophyll defect rates of FN treatment were like those of EMS (8.34-12.5\%) and higher than that of ${ }^{60} \mathrm{Co}(6.3 \%)$ in the $\mathrm{M}_{4}$ generation (Table 3). A side-by-side mutant comparison also indicated that EMS produced 12 collective putative mutants at $1.2 \%$ which is the highest amount of observable morphological mutations out of all populations (Table 3).

The discovery of a putative yellow mutant line FN 89 in the first trial that produced an albino phenotype in the second trial potentially indicates that the environment in which the plants are grown can affect chlorophyll biosynthesis and contribute to the severity of the phenotype observed. There were eight putative mutants scored in the $\mathrm{M}_{4}$ Katy population exposed to EMS at $0.4 \%$. This is significantly lower than the proposed number of mutants exposed to $1.2 \%$ EMS and may be due to higher concentrations corresponding to higher mutation effects in $\mathrm{M}_{4}$. Lower concentration mutagens overall yielded a smaller amount of morphological putative mutants likely due to a decreased probability of mutations being expressed. ${ }^{60} \mathrm{Co}$ mutants at 200 Gy were noticeably unhealthy due to the lethality of the radiation itself. The high amount of EMS mutants, with overall lower lethality, may be due to the point and base-pair mutations eliciting less lethal mutations than treatments that are more likely to cause small or large deletions. These data are corroborated by Li et al. [14] and Parry et al. [8] in proportion with one another with a high correlation and low variation.

One of the largest uncontrolled variables is that different loads of top soils with different nutrient levels were used for different trials. Additionally, different trials were performed from Sept 2017 to May 2018, so lighting conditions, humidity and temperature were not homogenous for all trials. Finally, human error was also possible because the determination of morphological mutations relies upon the observation of the scientist. The experiments in the first trial all yielded different numbers of morphological mutants. For instance, three $0.4 \%$ EMS mutants were discovered in the first trial and six $0.4 \%$ EMS were discovered in the second trial. Therefore, it may not be presumed that a similar number of mutants will occur under each trial. After two weeks post-planting in the first trial, it was obvious that there were an abnormal number of yellow mutants specifically in the FN mutants that is not consistent with the mutation effects discovered in other studies [10,14]. These abnormalities turned out not to be due to mutations, but rather due to suboptimal growth 
conditions. In addition, the seeds which were harvested in 2006 [25] may have lost health and viability in storage. The mutation frequencies should not be treated as the probability of any particular gene mutating because that assumption is dependent on the prevalence of the gene in the genome and its redundancy. However, the rate is an indicator of the probability of discovering the gene in relation to other forms of mutagens and the probability of this mutation expressing itself phenotypically. Overall, it can be inferred that EMS is the most effective mutagen according to the frequency of the morphological mutations observed in the $1.2 \%$ EMS population. However, each line will also be likely to carry other additional mutations elsewhere in the genome as well.

$\mathrm{M}_{2}$ plants are often used to identify mutants because it allows the widest selection of traits. However, the $\mathrm{M}_{2}$ plants are used in one trial because maintaining subsequent generations for some traits in this case, putative morphological mutants may be challenging. It was noticeable that mutation effects of FN treatments at $\mathrm{M}_{2}$ are inconsistent with that of $\mathrm{M}_{4}$ (Tables 2 and 3). However, the usage of $\mathrm{M}_{4}$ mutants in this study allowed two duplicate trials that uncovered additional mutations (Table 3). Most non-lethal heritable mutations may have gone to homozygosity by the $\mathrm{M}_{4}$ generation while homozygous lethal mutations would likely still be maintained in the heterozygous state. Therefore, it is recommended that the $\mathrm{M}_{4}$ be used for screening for mutants involved in disease resistance, yield, and quality.

\section{CONCLUSIONS}

In summary, a large mutant population consisting of 23,558 lines of Katy rice was developed and characterized. This population has been useful for identifying signaling components of blast pathogen (Magnaporthe oryzae) resistance [3,18,24] and is expected to be useful for other functional genomics studies and for the identification of agronomically important mutants for rice crop improvement. The Katy mutant population is being maintained and distributed through the USDA ARS Genetic Stocks-Oryza (GSOR) collection at Stuttgart, AR. Limited amounts of seed (ca. four seeds of each line) may be obtained through the Dale Bumpers National Rice Research Center, GSOR, USDA-ARS, 2890 HWY, 130 East, Stuttgart, 72160 AR (www.ars.usda.gov/GSOR). Requests from outside the USA must be accompanied by a seed import permit. Seeds are available for research purposes, including development and commercialization of new cultivars. If these genetic stocks contribute to the advancement of science or development of new cultivars it is requested that appropriate recognition be given to the source.

\section{SUPPLEMENTARY MATERIALS}

The following supplementary materials are available online at https://doi.org/10.20900/cbgg20190014, Supplementary Table S1, Genotypical evaluation of Katy mutants with diagnostic SSR markers 1. 


\section{AUTHOR CONTRIBUTIONS}

YJ and KM conceived; JNR reviewed project plan, assisted gamma irradiation; YJ, ZW, MHJ, and KM participated; YJ and MHJ wrote the paper; and all coauthors approve authorship and content.

\section{CONFLICTS OF INTEREST}

The authors declare that they have no conflicts of interest.

\section{FUNDING}

This work was funded in part by USDA ARS, Arkansas Rice Research and Promotion board to Jia, and the International Atomic Energy Agency.

\section{ACKNOWLEDGMENTS}

The authors thank the University of Arkansas Rice Research and Promotion Board and The International Atomic Energy Agency (IAEA) for the financial support and thank Andrew Blas, Lori Imboden, Tracy Bianco, Tony Beaten, Heather Box, Michael Lin, and Lorie Bernhardt of the United States Department of Agriculture-Agricultural Research Service Dale Bumpers National Rice Research Center (DB NRRC), Pratibha Singh, Jiankun Xie, Andrew Gibbons, Lin Wu of the University of Arkansas Rice Research and Extension Center, Dianxing Wu (IAEA fellow) of Zhejiang University, Hangzhou 310058, China, and Md. Ali Azam (IAEA fellow) of Bangladesh Institute of Nuclear Agriculture, Mymensingh Sadar 2200, Bangladesh for excellent technical assistance. A portion of this project was accomplished through a science fair project of Mary S. Jia of Applied Research Methods class under the supervision of Brian Monson at Arkansas School for Mathematics, Sciences and the Arts (ASMSA), Hot Springs, AR 71901, USA. Specifically, we appreciate that Mary S. Jia collected, analyzed, interpreted the $\mathrm{M}_{4}$ data and prepared the first draft of the manuscript under supervision of Monson for her class at ASMSA. The USDA is an equal opportunity provider and employer.

\section{REFERENCES}

1. Jiang Y, Cai Z, Xie W, Long T, Yu H, Zhang Q. Rice functional genomics research: progress and implications for crop genetic improvement. Biotechnol Adv. 2012;30:1059-70.

2. Li Y, Xiao J, Chen L, Huang X, Cheng Z, Han B, et al. Rice functional genomic research: Past decade and future. Mol Plant. 2018;11:359-80.

3. Zhao H, Wang X, Jia Y, Minkenberg B, Wheatley M, Fan J, et al. The rice blast resistance gene Ptr encodes an atypical protein required for broad-spectrum disease resistance. Nat Commun. 2018;9:2039.

4. Liu G, Jia Y, Correa-Victoria F, Prado GA, Yeater KM, McClung A, et al. Mapping quantitative trait loci responsible for resistance to sheath blight in rice. Phytopathology 2009;99:1078-84. 
5. Venu RC, Sreerekha MV, Madhav MS, Nobuta K, Mohan KM, Chen S, et al. Deep transcriptome sequencing reveals the expression of key functional and regulatory genes involved in the abiotic stress signaling pathways in rice. J Plant Biol. 2013;56:216-31.

6. Chodavarapu RK, Feng S, Ding B, Simon SA, Lopez D, Jia Y, et al. Transcriptome and methylome interactions in rice hybrids. Proc Natl Acad Sci U S A. 2012;109:12040-5.

7. Caicedo AL, Williamson SH, Hernandez SH, Boyko A, Fledel-Alon A, York TL, et al. Genome-wide patterns of nucleotide polymorphism in domesticated rice. PLoS Genet. 2007;3:e163.

8. Parry MA, Madgwick PJ, Bayon C, Tearall K, Hernandez-Lopez A, Rakszegi M, et al. Mutant discovery for crop improvement. J Exp Bot. 2009;60:2817-25.

9. Talebi ALB, Talebi AMB, Shahrokhifar B. Ethyl methane sulphonate (EMS) induced mutagenesis in Malaysian rice (cv. MR219) for lethal dose determination. Am J Plant Sci. 2012;3:1661-5.

10. Till BJ, Cooper J, Tai TH, Colowit P, Greene EA, Henikoff S, et al. Discovery of chemically induced mutations in rice by TILLING. BMC Plant Biol. 2007;7:19.

11. Morita R, Kusaba M, Iida S, Yamaguchi H, Nishio T, Nishimura M. Molecular characterization of mutations induced by gamma irradiation in rice. Genes Genet Syst. 2009;84:361-70.

12. Li X, Lassner M, Zhang Y. Deleteagene: a fast neutron deletion mutagenesisbased gene knockout system for plants. Comp Funct Genomics. 2002;3:158-60.

13. Jia Y. Artificial introgression of a large chromosome fragment around the rice blast resistance gene $\mathrm{Pi}$-ta in backcross progeny and several elite rice cultivars. Heredity. 2009;103:333-9.

14. McCallum CM, Comai L, Greene EA, Henikoff S. Targeting Induced Local Lesions IN Genomes (TILLING) for plant functional genomics. Plant Physiol. 2000;123:439-42.

15. Moldenhauer KAK, Lee FN, Norman RJ, Helms RS, Wells BR, Dilday RH, et al. Registration of 'Katy’ rice. Crop Sci. 1990;30:747-8.

16. Chao CT, Moldenhauer KAK, Ellingboe AH. Genetic analysis of resistance/susceptibility in individual $\mathrm{F}_{3}$ families of rice against strains of Magnaporthe grisea containing different genes for avirulence. Euphytica. 1999;109:183-90.

17. Wang X, Lee S, Wang J, Ma J, Bianco TA, Jia Y. Current advances on genetic resistance to rice blast disease. In: Yan W, Bao J, editors. Rice-Germplasm, Genetics and Improvement. London (UK): Intechopen Limited; 2014. p. 195-217.

18. Jia Y, Martin R. Identification of a new locus $\operatorname{Ptr}(t)$ required for rice blast resistance gene $\mathrm{Pi}$-ta-mediated resistance. Mol Plant Microbe Interact. 2008;21:396-403.

19. Hu J, Rutger JN. Pollen characteristics and genetics of induced and spontaneous genetic male sterile mutants in rice. Plant Breed. 1992;109:97-107.

20. Xin Z, Velten JP, Oliver MJ, Burke JJ. High-throughput DNA extraction method suitable for PCR. BioTechniques. 2003;34:802-26. 
21. Garris AJ, Tai T, Coburn J, Kresovich S, McCouch SR. Genetic structure and diversity in Oryza sativa L. Genetics. 2005;169:1631-8.

22. Agrama HA, Yan W, Jia MH, Fjellstrom RG, McClung AM. Genetic structure associated with diversity and geographic distribution in the USDA rice world collection. Nat Sci. 2010;2:247-91.

23. Karasov TL, Chae E, Herman JJ, Bergelson J. Mechanisms to mitigate the tradeoff between growth and defense. Plant Cell. 2017;29:666-80.

24. Jia Y. Registration of Katy lesion mimic mutant 1. Crop Sci. 2005;45:1675.

25. Jia Y, Xie J, Rutger JN. Development and characterization of Katy deletion mutant populations for functional genomics of host-parasite interactions and rice improvement. Plant Mutation Rep. 2006;1:143-7.

How to cite this article:

Jia Y, Wang Z, Jia MH, Rutger JN, Moldenhauer KAK. Development and Characterization of a Large Mutant Population of a Rice Variety Katy for Functional Genomics Studies and Breeding. Crop Breed Genet Genom. 2019;1:e190014. https://doi.org/10.20900/cbgg20190014 